\title{
Control-to-output Transfer Function of the Open-loop Step-up Converter in CCM Operation
}

\author{
Faqiang Wang ${ }^{\dagger}$ and Xikui Ma*
}

\begin{abstract}
Based on the average method and the geometrical technique to calculate the average value, the average model of the open-loop step-up converter in CCM operation is established. The DC equilibrium point and corresponding small signal model is derived. The control-to-output transfer function is presented and analyzed. The theoretical analysis and PSIM simulations shows that the control-to-output transfer function includes not only the DC input voltage and the DC duty cycle, but also the two inductors, the two energy-transferring capacitors, the switching frequency and the load. Finally, the hardware circuit is designed, and the circuit experimental results are given to confirm the effectiveness of theoretical derivations and analysis.
\end{abstract}

Keywords: Step-up converter, Average model, Small signal model, Control-to-output transfer function

\section{Introduction}

As one of the important research area in the filed of DCDC power supply, exploring a new topology of DC-DC converter to obtain the good performance in practical engineering is very meaningful and valuable work and many good results have been presented [1-12]. Among of them, a novel step-up converter, which combining the KY converter and the traditional Buck-Boost converter, has been proposed by K. I. Hwu et al in 2011 to boost the low input voltage to the high output voltage [5]. Comparing to the existing DC-DC boosting converter [7-16], this novel step-up converter has some outstanding advantages [5]. For example, by comparing to the traditional Boost converter [7], this novel step-up converter has no pulsating current through the output capacitor since there is an output inductor, thereby causing the output voltage ripple to be small [5].

By comparing to the KY converter whose voltage conversion ratio up to two [8], the voltage converter ratio of this novel step-up converter can be up to infinite. By comparing to the DC-DC boosting converters that include the coupled inductors with turn ratio [9-10], there is no leakage inductance should be considered in this novel step-up converter. Also, by comparing to the DC-DC boosting converters whose power switch is floating and need the isolated driving signal [11-12], it is easy to drive this novel step-up converter since no isolated gate driver is needed instead of one half-bridge gate driver. Accordingly,

$\dagger \quad$ Corresponding Author: State Key Laboratory of Electrical Insulation and Power Equipment, School of Electrical Engineering, Xi'an Jiaotong University, Xi'an, China. (eecjob@126.com).

* State Key Laboratory of Electrical Insulation and Power Equipment, School of Electrical Engineering, Xi'an Jiaotong University, Xi'an, China.

Received: October 6, 2013 ; Accepted: March 1, 2014 it can be believed that this novel step-up converter will be widely accepted and used in the area of boosting the low input voltage to the high output voltage to obtain enough output power and voltage amplitude, such as uninterruptible power supplies, solar cell powering systems, battery powering systems, and so on, to instead of the traditional Boost converter and the voltage boosting converter which uses the coupling or needs the isolated gate driver to obtain the high output voltage in the future. Therefore, it is necessary to establish the accurate model for this novel step-up converter to prepare for consequent designing.

However, up to now, there are only a few studies to do this point. In [5], under the assumption that the two energy-transferring capacitors were large enough to keep the voltage across themselves at some values, the formulas for DC output voltage which only includes the DC duty cycle and the DC input voltage has been derived. But, the absence of the average model and corresponding small signal model of the open-loop step-up converter in [5] leads to hard to design the controller to obtain the good performance to satisfy the requirements in practical engineering. In other words, establishing the average model and small signal model of the open-loop step-up converter is the key and important step for consequent designing and analysis.

The rest of the paper is organized as follows. In section 2, the circuit operation, the mathematical model and some PSIM simulations of the open-loop step-up converter in CCM operation are given. Then, the average model is established. The DC equilibrium point and the controlto-output transfer function are derived and analyzed. In section 4, the circuit experiments are given for confirmation. Finally, some concluding remarks are given in section 5 . 


\section{Circuit Operation, Mathematical Model and PSIM Simulations}

The circuit schematic of this step-up converter is shown in Fig. 1. It can be seen that this step-up converter consists of the input voltage $v_{i n}$, two inductors: $L_{1}$ and $L_{2}$, two energy-transferring capacitors: $C_{1}$ and $C_{2}$, two power switches: $\mathrm{S}_{1}$ and $\mathrm{S}_{2}$, one diode $\mathrm{D}_{1}$, one output capacitor $C_{0}$ and one load $R$.

The switches $S_{1}$ and $S_{2}$ are driven by one half-bridge gate driver with the switching frequency being $f$ and the DC duty cycle being $D$. The voltage across $C_{0}, C_{1}$ and $C_{2}$ are defined as $v_{0}, v_{1}$ and $v_{2}$, respectively. The current through the inductor $L_{1}$ and $L_{2}$ are defined as $i_{L 1}$ and $i_{L 2}$, respectively. The current through $C_{1}$ and $C_{2}$ are defined as $i_{C 1}$ and $i_{C 2}$, respectively. Note that, here, only the continuous conduction mode (CCM) operation is concerned, i.e., there are only two operation modes in this novel step-up converter, which are shown in Figs. 2(a) and (b), respectively. Additionally, the circuit parameters here are chosen as $v_{i n}=6 \mathrm{~V}, C_{1}=2 \mu \mathrm{F}, C_{2}=4.7 \mu \mathrm{F}, L_{1}=3 \mathrm{mH}, L_{2}=1 \mathrm{mH}$,

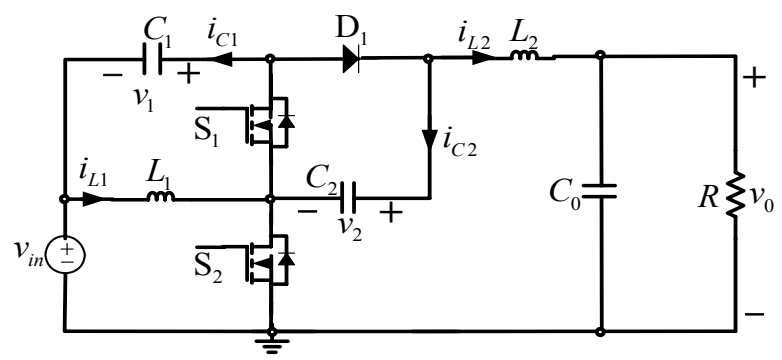

Fig. 1. The circuit schematic of step-up converter

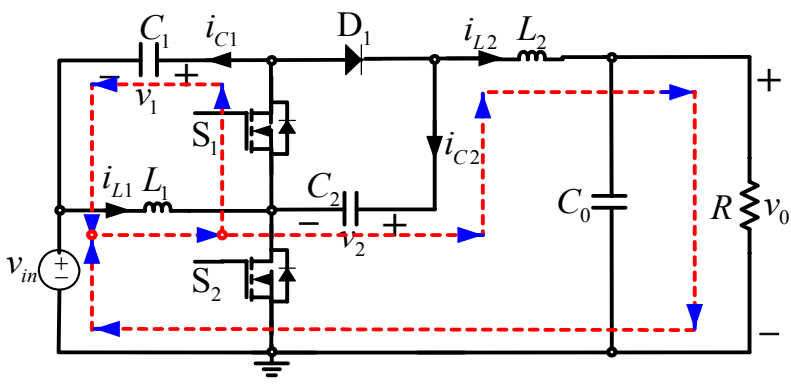

(a)

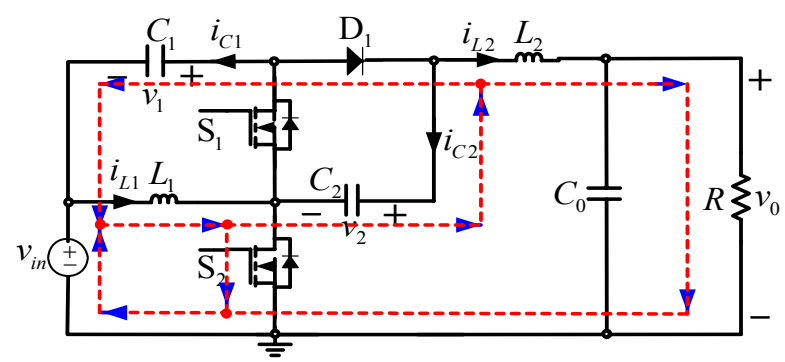

(b)

Fig. 2. The power flows of the two operation modes of step-up converter: (a) mode 1; (b) mode 2.
$C_{0}=40 \mu \mathrm{F}, R=80 \Omega, G=1 / R, f=25 \mathrm{kHz}, T=1 / f$, and $D=0.5$.

Mode 1: the power switch $\mathrm{S}_{1}$ is turned on whereas $\mathrm{S}_{2}$ is turned off and the diode $\mathrm{D}_{1}$ is not conducted for its inversebiased voltage. The power flows for this mode are shown in Fig. 2(a). According to the circuit theory, the equations for describing this mode can be derived as follows

$$
\left\{\begin{array}{l}
\frac{d i_{L 1}}{d t}=-\frac{v_{1}}{L_{1}} \\
\frac{d i_{L 2}}{d t}=\frac{v_{i n}+v_{1}+v_{2}-v_{0}}{L_{2}} \\
\frac{d v_{2}}{d t}=-\frac{i_{L 2}}{C_{2}} \\
\frac{d v_{0}}{d t}=\frac{i_{L 2}}{C_{0}}-\frac{v_{0}}{R C_{0}} \\
i_{C 1}=i_{L 1}-i_{L 2}
\end{array}\right.
$$

Mode 2: the power switch $\mathrm{S}_{1}$ is turned off whereas $\mathrm{S}_{2}$ is turned on and the diode $\mathrm{D}_{1}$ is conducted for its forwardbiased voltage. The power flows for this mode are shown in Fig. 2(b). According to the circuit theory, the equations for describing this mode can also be derived as follows

$$
\left\{\begin{array}{l}
\frac{d i_{L 1}}{d t}=\frac{v_{i n}}{L_{1}} \\
\frac{d i_{L 2}}{d t}=\frac{v_{i n}+v_{1}-v_{0}}{L_{2}} \\
\frac{d v_{2}}{d t}=-\frac{i_{L 2}+i_{C 1}}{C_{2}} \\
\frac{d v_{0}}{d t}=\frac{i_{L 2}}{C_{0}}-\frac{v_{0}}{R C_{0}} \\
v_{1}=v_{2}-v_{i n}
\end{array}\right.
$$

Based on the PSIM software which is widely used to simulate the motor and the power electronics $[17,18]$, the PSIM simulations for the voltage $v_{1}+v_{i n}, v_{2}$ and the PWM signal $v_{d}$ are shown in Fig. 3.

From the time-domain waveform of the voltage $v_{1}+v_{i n}$ in Fig. 3(a) and the voltage $v_{2}$ in Fig. 3(b), it is easily obtained that the ripple of the voltage $v_{1}+v_{i n}$ is within $(9.9 \mathrm{~V}, 13.8 \mathrm{~V})$ and the voltage $v_{2}$ is within $(9.1 \mathrm{~V}, 10.5 \mathrm{~V})$. Thus, both the ripples of the voltage $v_{1}+v_{\text {in }}$ and $v_{2}$ can not be considered as being equal to zero when calculating their average values since their ripples are not small enough. In other words, in [5], the assumption that the two energy-transferring capacitors are large enough to 

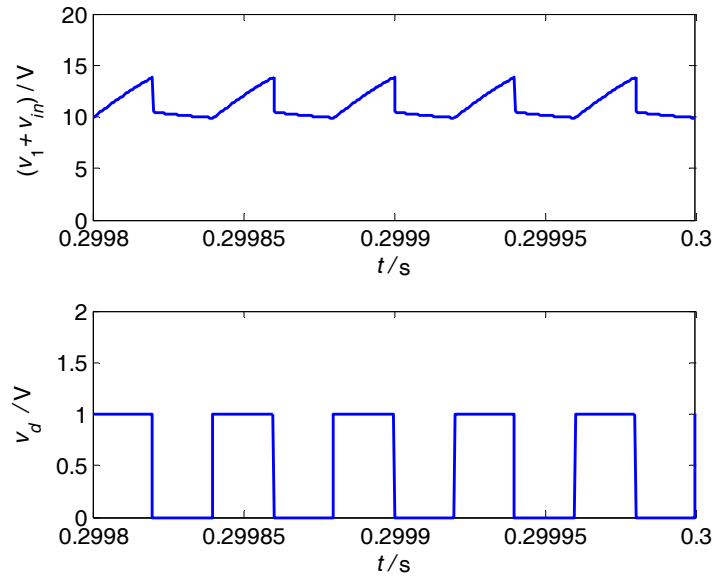

(a)
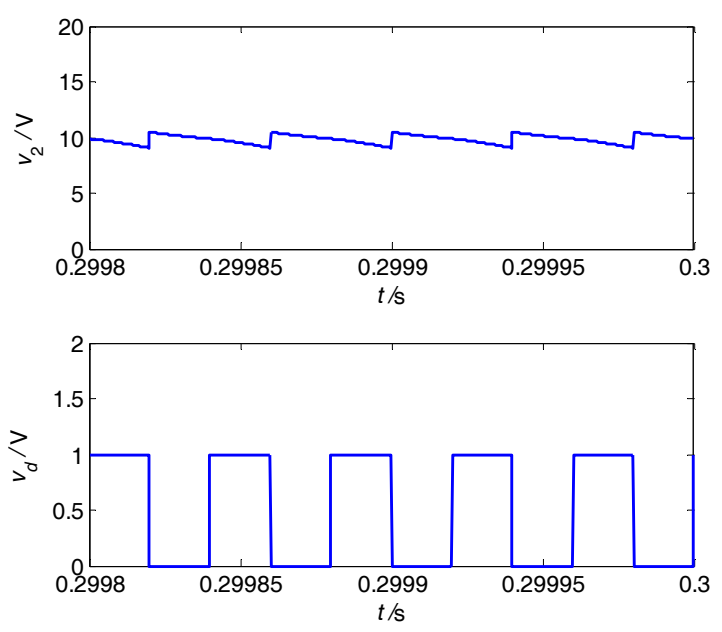

(b)

Fig. 3. PSIM simulations: (a) the voltage $v_{1}+v_{\text {in }}$ and the PWM signal $v_{d}$; (b) the voltage $v_{2}$ and the PWM signal $v_{d}$.

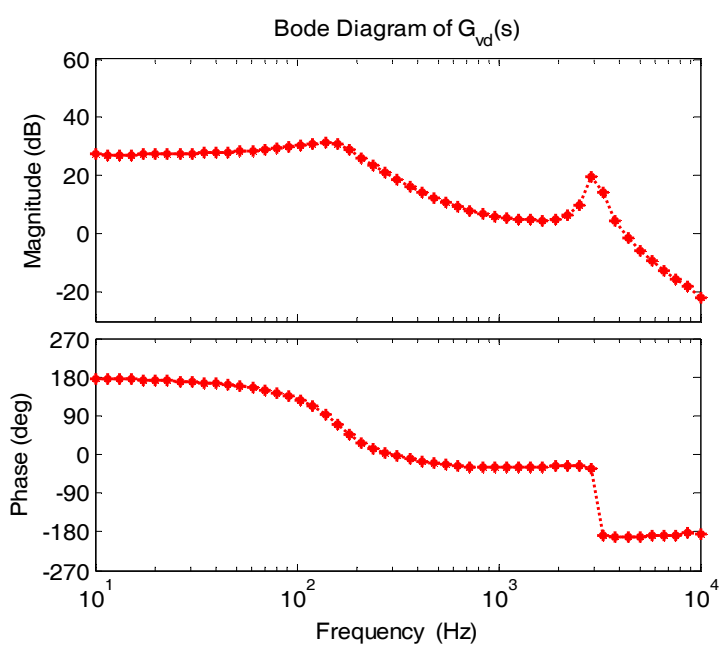

Fig. 4. Bode diagram of $G_{v d}(s)$ from PSIM simulations

keep the voltage across themselves being constant at some values is only an extreme case, i.e., the assumption in [5] can not be satisfied in some cases in practical engineering.

Also, from the characteristics of PSIM software [19-20], i.e., bode diagram of switching power converter can be directly obtained by using its switch mode form, bode diagram of the control-to-output transfer function $\left(G_{v d}(s)\right)$ of this novel step-up converter can be obtained, which is shown in Fig. 4. These PSIM simulations will be used to preliminary confirm the effectiveness of the theoretical derivations and analysis in the following sections.

\section{Theoretical Derivations and Analysis}

Based on the average method [21] and the Eqs. (1) and (2), the average model of this open-loop step-up converter can be easily obtained as follows

$$
\left\{\begin{array}{l}
\frac{d\left\langle i_{L 1}\right\rangle}{d t}=-\frac{\left\langle v_{1}\right\rangle}{L_{1}} d+\frac{\left\langle v_{i n}\right\rangle}{L_{1}}(1-d) \\
\frac{d\left\langle i_{L 2}\right\rangle}{d t}=\frac{\left\langle v_{i n}\right\rangle+\left\langle v_{1}\right\rangle-\left\langle v_{0}\right\rangle}{L_{2}}+\frac{\left\langle v_{2}\right\rangle}{L_{2}} d \\
\frac{d\left\langle v_{2}\right\rangle}{d t}=-\frac{\left\langle i_{L 2}\right\rangle}{C_{2}} d-\frac{\left\langle i_{L 2}\right\rangle+\left\langle i_{C 1}\right\rangle}{C_{2}}(1-d) \\
\frac{d\left\langle v_{0}\right\rangle}{d t}=\frac{\left\langle i_{L 2}\right\rangle}{C_{0}}-\frac{\left\langle v_{0}\right\rangle}{R C_{0}}
\end{array}\right.
$$

where $\left\langle i_{L 1}\right\rangle,\left\langle i_{L 2}\right\rangle,\left\langle i_{C 1}\right\rangle,\left\langle v_{0}\right\rangle,\left\langle v_{1}\right\rangle,\left\langle v_{2}\right\rangle$ and $\left\langle v_{i n}\right\rangle$ are the corresponding average value of $i_{L 1}, i_{L 2}, i_{C 1}, v_{0}, v_{1}, v_{2}$ and $v_{i n}$, respectively, and $d$ is the duty cycle.

Obviously, in order to derive the average model of this open-loop step-up converter completely, the expressions for $\left\langle v_{1}\right\rangle$ and $\left\langle i_{C 1}\right\rangle$ must be derived. Here, for convenience to derive the expression for $\left\langle v_{1}\right\rangle$, the time-domain waveforms of the voltage $v_{1}+v_{i n}, v_{2}$ and the PWM signal $v_{d}$ are replotted in Fig. 5.

In Fig. 5, it is clearly seen that the voltage $v_{1}+v_{\text {in }}$ is the same as the voltage $v_{2}$ within $((N+d) T,(N+1) T)$. However, they are different from each other within $(N T,(N+d) T)$. Also, one can see that there are jumps on the voltage across the two energy-transferring capacitors since they are abruptly changing at $(N+d) T$, respectively. Therefore, this novel step-up converter is very different from the traditional DC-DC converters which have no jumps on the voltage across the capacitors, such as Cuk or Sepic converters. Thus, unlike establishing average model and small signal model for Cuk or Sepic converters which using the average method directly, the jumps on the voltage across the two energy-transferring capacitors in this novel step-up converter must be firstly considered and calculated by using the geometrical technique.

Here, the voltage $v_{1}+v_{i n}$ and $v_{2}$ are all denoted as $V_{N 0}$ after abruptly changing at $(N+d) T$ and $V_{N+1}$ at $(N+1) T$, respectively. Also, they are denoted as $V_{N 1}$ and $V_{N 2}$ before 


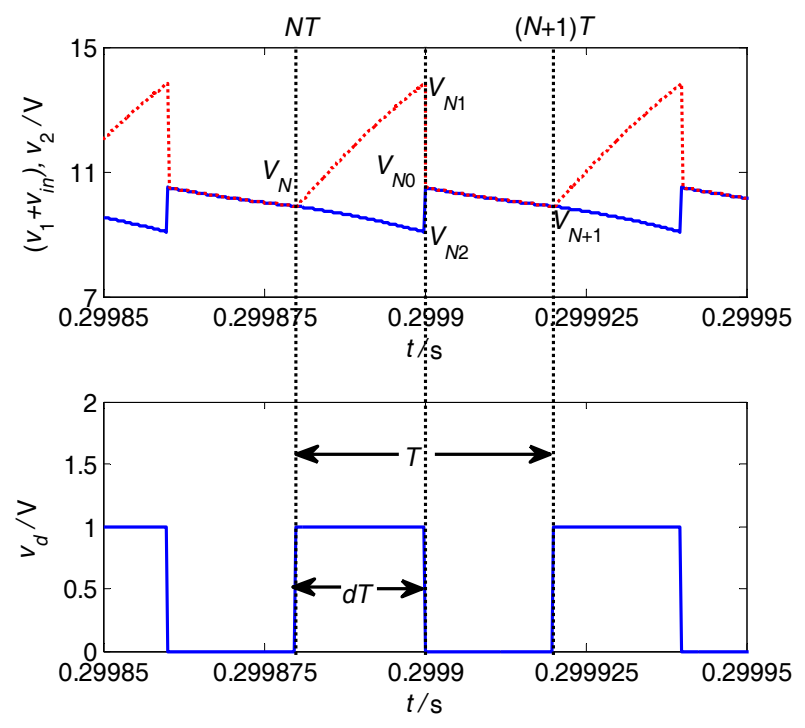

Fig. 5. The re-plotted of the voltage $v_{1}+v_{\text {in }}$ (dashed line), $v_{2}$ (solid line) and the PWM signal $v_{d}$

abruptly changing at $(N+d) T$, respectively, and $V_{N}$ at $N T$. Note that $V_{N}=V_{N+1}$. Moreover, assuming that the voltage $v_{1}+v_{\text {in }}$ decreases linearly within $((N+d) T,(N+1) T)$ and increases linearly within $(N T,(N+d) T)$, and the voltage $v_{2}$ decreases linearly within $(N T,(N+d) T)$ and $((N+d) T$, $(N+1) T)$. Based on the geometrical technique to calculate the average value in the field of power converter and Fig. 5 , the following formulas can be obtained

$$
\begin{gathered}
\left(\left\langle v_{1}\right\rangle+\left\langle v_{\text {in }}\right\rangle\right) T=\frac{V_{N}+V_{N 1}}{2} d T+\frac{V_{N 0}+V_{N}}{2}(1-d) T \\
\left\langle v_{2}\right\rangle T=\frac{V_{N}+V_{N 2}}{2} d T+\frac{V_{N 0}+V_{N}}{2}(1-d) T
\end{gathered}
$$

where

$$
\begin{gathered}
V_{N 1}=V_{N}+\frac{\left\langle i_{L 1}\right\rangle-\left\langle i_{L 2}\right\rangle}{C_{1}} d T \\
V_{N 2}=V_{N}-\frac{\left\langle i_{L 2}\right\rangle}{C_{2}} d T
\end{gathered}
$$

Thus, the expression for $\left\langle v_{1}\right\rangle$ can be derived by making (4) minus (5) and the result is shown as follows

$$
\left\langle v_{1}\right\rangle=\left\langle v_{2}\right\rangle+\alpha\left\langle i_{L 2}\right\rangle d^{2}+\beta\left\langle i_{L 1}\right\rangle d^{2}-\left\langle v_{i n}\right\rangle
$$

where $\alpha=\left(C_{1}-C_{2}\right) T /\left(2 C_{1} C_{2}\right)$ and $\beta=T /\left(2 C_{1}\right)$.

In addition, based on the ampere-second balance, $\left\langle i_{C 1}\right\rangle$ can be expressed as a function of $\left\langle i_{L 1}\right\rangle$ and $\left\langle i_{L 2}\right\rangle$, given by

$$
\left\langle i_{C 1}\right\rangle=\left(\left\langle i_{L 2}\right\rangle-\left\langle i_{L 1}\right\rangle\right) \frac{d}{1-d}
$$

Therefore, the average model of the open-loop step-up converter can be obtained by taking (8) and (9) into (3), and its expression is

$$
\left\{\begin{array}{l}
\frac{d\left\langle i_{L 1}\right\rangle}{d t}=-\frac{\left\langle v_{2}\right\rangle}{L_{1}} d-\frac{\alpha}{L_{1}}\left\langle i_{L 2}\right\rangle d^{3}-\frac{\beta}{L_{1}}\left\langle i_{L 1}\right\rangle d^{3}+\frac{\left\langle v_{i n}\right\rangle}{L_{1}} \\
\frac{d\left\langle i_{L 2}\right\rangle}{d t}=-\frac{\left\langle v_{0}\right\rangle}{L_{2}}+\frac{\alpha}{L_{2}}\left\langle i_{L 2}\right\rangle d^{2}+\frac{\beta}{L_{2}}\left\langle i_{L 1}\right\rangle d^{2}+\frac{\left\langle v_{2}\right\rangle}{L_{2}}(1+d) \\
\frac{d\left\langle v_{2}\right\rangle}{d t}=-\frac{\left\langle i_{L 2}\right\rangle(1+d)-\left\langle i_{L 1}\right\rangle d}{C_{2}} \\
\frac{d\left\langle v_{0}\right\rangle}{d t}=\frac{\left\langle i_{L 2}\right\rangle}{C_{0}}-\frac{\left\langle v_{0}\right\rangle}{R C_{0}}
\end{array}\right.
$$

The DC equilibrium point and the small signal model of (10) can be derived by using the perturbation and linearization of (10).

Assuming that $I_{L 1}, I_{L 2}, V_{0}, V_{2}, V_{\text {in }}$ and $D$ are the DC values of $\left\langle i_{L 1}\right\rangle,\left\langle i_{L 2}\right\rangle,\left\langle v_{0}\right\rangle,\left\langle v_{2}\right\rangle,\left\langle v_{i n}\right\rangle$ and $d$, respectively, and $\hat{i}_{L 1}, \hat{i}_{L 2}, \hat{v}_{0}, \hat{v}_{2}, \hat{v}_{i n}$ and $\hat{d}$ are their small ac values. Additionally, the following equations are assumed

$$
\left\{\begin{array} { l } 
{ \langle i _ { L 1 } \rangle = I _ { L 1 } + \hat { i } _ { L 1 } } \\
{ \langle i _ { L 2 } \rangle = I _ { L 2 } + \hat { i } _ { L 2 } } \\
{ \langle v _ { 0 } \rangle = V _ { 0 } + \hat { v } _ { 0 } } \\
{ \langle v _ { 2 } \rangle = V _ { 2 } + \hat { v } _ { 2 } } \\
{ \langle v _ { i n } \rangle = V _ { i n } + \hat { v } _ { i n } } \\
{ d = D + \hat { d } }
\end{array} \quad \text { with } \quad \left\{\begin{array}{l}
\hat{i}_{L 1} \square I_{L 1} \\
\hat{i}_{L 2} \square I_{L 2} \\
\hat{v}_{0} \square V_{0} \\
\hat{v}_{2} \square V_{2} \\
\hat{v}_{i n} \square V_{i n} \\
\hat{d} \square D
\end{array}\right.\right.
$$

Thus, the DC equilibrium point of the open-loop step-up converter can be obtained by substituting (11) into (10) and then separating the DC values out. The result is

$$
\left\{\begin{array}{l}
I_{L 1}=\frac{V_{i n} G(1+D)^{2}}{D^{2}+\alpha G D^{5}+\beta G D^{4}(1+D)} \\
I_{L 2}=\frac{V_{\text {in }} G(1+D)}{D+\alpha G D^{4}+\beta G D^{3}(1+D)} \\
V_{2}=V_{\text {in }} \frac{1-\alpha G D^{2}-\beta G(1+D) D}{D+\alpha G D^{4}+\beta G D^{3}(1+D)} \\
V_{0}=\frac{V_{\text {in }}(1+D)}{D+\alpha G D^{4}+\beta G D^{3}(1+D)}
\end{array}\right.
$$

where $G=1 / R$. From the formula for DC output voltage, i.e., $V_{0}$, it is easily seen that its expression includes not only the DC input voltage $V_{\text {in }}$ and the DC duty cycle $D$, but also the two energy-transferring capacitors $C_{1}$ and $C_{2}$, the switching frequency $f$ and the load $R$.

By calculating the voltage $V_{0}$ over the voltage $V_{i n}$, the voltage conversion ratio can also be derived, which is shown as follows 


$$
\frac{V_{0}}{V_{\text {in }}}=\frac{1+D}{D+\alpha G D^{4}+\beta G D^{3}(1+D)}
$$

It is obvious that the voltage conversion ratio here is very different from the result in [5] which only includes the DC duty cycle $D$.

Moreover, from (1), (2), (12) and Fig. 5, the current ripples $\left(\Delta i_{L 1}\right.$ and $\left.\Delta i_{L 2}\right)$ of two inductors $\left(L_{1}\right.$ and $\left.L_{2}\right)$ and the voltage ripples $\left(\Delta v_{1}, \Delta v_{2}\right.$ and $\left.\Delta v_{0}\right)$ of three capacitors $\left(C_{1}, C_{2}\right.$ and $\left.C_{0}\right)$ can be derived, which are shown as follows. In other words, if these current ripples and voltage ripples, the DC duty cycle $D$, the $\mathrm{DC}$ input voltage $V_{i n}$, the load $R(G=1 / R)$ and the switching period $T$ have already been given, the values of $L_{1}, L_{2}, C_{1}, C_{2}$ and $C_{0}$ can be calculated and these are very helpful for designing these parameters to satisfy the requirements in practical engineering.

$$
\left\{\begin{array}{l}
\Delta i_{L 1}=\frac{V_{\text {in }}(1-D) T}{L_{1}} \\
\Delta i_{L 2}=\frac{(\alpha G D+\beta G(1+D)+1)(1-D) V_{\text {in }} T}{\left(1+\alpha G D^{3}+\beta G D^{2}(1+D)\right) L_{2}} \\
\Delta v_{1}=\frac{V_{\text {in }} G(1+D) T}{\left(D+\alpha G D^{4}+\beta G D^{3}(1+D)\right) C_{1}} \\
\Delta v_{2}=\frac{\left(C_{1} D T+C_{2} T\right) V_{i n} G(1+D)}{\left(C_{1}+C_{2}\right) C_{2}\left(D+\alpha G D^{4}+\beta G D^{3}(1+D)\right)} \\
\Delta v_{0}=\frac{(\alpha G D+\beta G(1+D)+1)(1-D) V_{i n} T^{2}}{8 C_{0}\left(1+\alpha G D^{3}+\beta G D^{2}(1+D)\right) L_{2}}
\end{array}\right.
$$

Furthermore, the small signal model of the open-loop step-up converter can be derived by substituting (11) into (10) and then separating the small ac variations out and omitting the second and higher order terms since their values are very small. The result is

$$
\left\{\begin{array}{l}
\frac{d \hat{i}_{L 1}}{d t}=\frac{\hat{v}_{i n}-D \hat{v}_{2}-\left(V_{2}+3 \alpha D^{2} I_{L 2}+3 \beta D^{2} I_{L 1}\right) \hat{d}-\beta D^{3} \hat{i}_{L 1}-\alpha D^{3} \hat{i}_{L 2}}{L_{1}} \\
\frac{d \hat{i}_{L 2}}{d t}=\frac{(1+D) \hat{v}_{2}-\hat{v}_{0}+\left(V_{2}+2 \alpha D I_{L 2}+2 \beta D I_{L 1}\right) \hat{d}+\beta D^{2} \hat{i}_{L 1}+\alpha D^{2} \hat{i}_{L 2}}{L_{2}} \\
\frac{d \hat{v}_{2}}{d t}=-\frac{\left(I_{L 2}-I_{L 1}\right) \hat{d}-D \hat{i}_{L 1}+(1+D) \hat{i}_{L 2}}{C_{2}} \\
\frac{d \hat{v}_{0}}{d t}=\frac{\hat{i}_{L 2}}{C_{0}}-\frac{\hat{v}_{0}}{R C_{0}}
\end{array}\right.
$$

Therefore, the control-to-output transfer function $\left(G_{v d}(s)\right)$ can be derived by using the Laplace transform on (15) and its definition, and the expression is

$$
G_{v d}(s)=\frac{b_{0} s^{2}+b_{1} s+b_{2}}{a_{0} s^{4}+a_{1} s^{3}+a_{2} s^{2}+a_{3} s+a_{4}}
$$

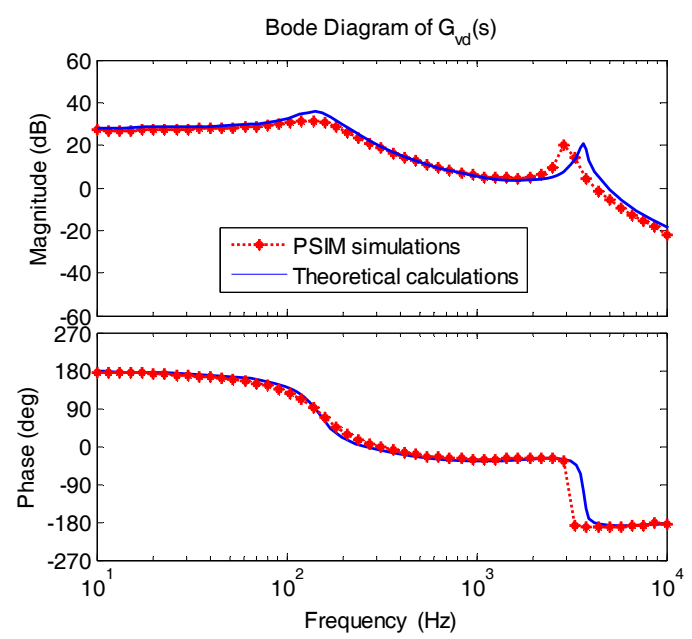

Fig. 6. Comparisons about bode diagram of $G_{v d}(s)$ between theoretical calculations and PSIM simulations

where

$\left\{\begin{array}{l}a_{0}=L_{2} C_{2} L_{1} C_{0} \\ a_{1}=L_{2} C_{2} L_{1} G+L_{2} C_{2} \beta D^{3} C_{0}-C_{2} L_{1} \alpha D^{2} C_{0} \\ a_{2}=L_{2} C_{2} \beta D^{3} G-C_{2} L_{1} \alpha D^{2} G+L_{2} D^{2} C_{0}+L_{1}(1+D)^{2} C_{0}+C_{2} L_{1} \\ a_{3}=L_{2} D^{2} G+L_{1}(1+D)^{2} G+\beta D^{4}(1+D) C_{0}+\alpha D^{5} C_{0}+C_{2} \beta D^{3} \\ a_{4}=\beta D^{4}(1+D) G+\alpha D^{5} G+D^{2} \\ b_{0}=\left(V_{2}+2 \alpha D I_{L 2}+2 \beta D I_{L 1}\right) C_{2} L_{1} \\ b_{1}=V_{2} C_{2} \beta\left(D^{3}-D^{2}\right)-\left(\alpha I_{L 2}+\beta I_{L 1}\right) C_{2} \beta D^{4}+\left(I_{L 1}-I_{L 2}\right)(1+D) L_{1} \\ b_{2}=\left(I_{L 1}-I_{L 2}\right) \beta D^{4}-V_{2} D-\alpha D^{3} I_{L 2}-\beta D^{3} I_{L 1}-3 \alpha I_{L 2} D^{4}-3 \beta I_{L 1} D^{4}\end{array}\right.$

From (16), it is obvious that the derived control-tooutput transfer function $G_{v d}(s)$ includes not only the DC input voltage $V_{\text {in }}$ and the DC duty cycle $D$, but also the two inductors: $L_{1}$ and $L_{2}$, the two energy-transferring capacitors: $C_{1}$ and $C_{2}$, the switching frequency $f$ and the load $R$.

Fig. 6 shows bode diagram of $G_{v d}(s)$ from the theoretical calculations. At the same time, for confirming the effectiveness of the derived $G_{v d}(s)$ preliminary, the PSIM simulations about bode diagram of $G_{v d}(s)$ are also replotted in Fig. 6. Obviously, the theoretical calculations are in good agreement with the PSIM simulations. Thus, the derived control-to-output transfer function $G_{v d}(s)$ here is an effective model for describing the small signal dynamical behaviors of the open-loop step-up converter.

\section{Circuit Experiments}

In order to confirm the effectiveness of the theoretical derivations and analysis further, the hardware circuit of the step-up converter is implemented by using MOSFET IRFP460 for realizing the switch $\mathrm{S}_{1}$ and $\mathrm{S}_{2}$, and MUR1560 


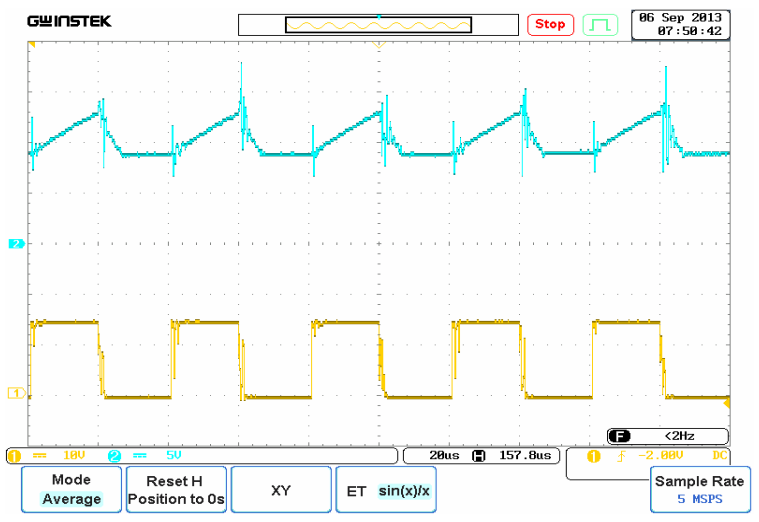

(a)

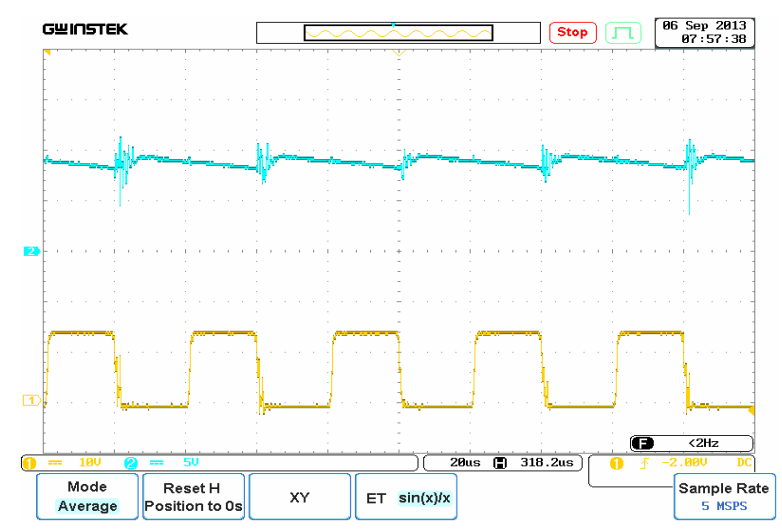

(b)

Fig. 7. Time-domain waveforms from the circuit experiments with time scale: $20 \mu \mathrm{s} /$ div: (a) $v_{1}+v_{i n}$ (upper: $5 \mathrm{~V} / \mathrm{div}$ ) and $v_{d}$ (lower: $10 \mathrm{~V} / \mathrm{div}$ ); (b) $v_{2}$ (upper: $5 \mathrm{~V} / \mathrm{div}$ ) and $v_{d}$ (lower: $10 \mathrm{~V} / \mathrm{div}$ ).

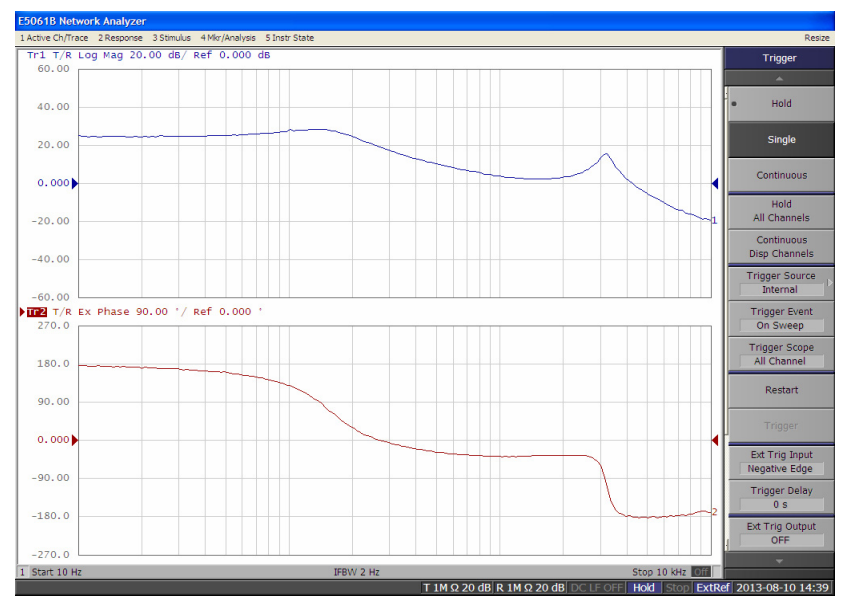

Fig. 8. Bode diagram of $G_{v d}(s)$ from the circuit experiments

for realizing the diode $\mathrm{D}_{1}$. The digital oscilloscope GDS 3254 is employed to capture the measured time-domain waveforms in the probes and the Agilent E5061B LF-RF network analyzer is employed to capture the measured gain and phase in the probes. Taking the circuit parameters as in section 2, the voltage $v_{1}+v_{i n}, v_{2}$ and the PWM signal $v_{d}$ from the circuit experiments are shown in Fig.
7. Comparing Fig. 7 with Fig. 3, it can be seen that they are in good agreement with each other. Therefore, it is confirmed that the voltage $v_{1}+v_{i n}$ is really not equal to the voltage $v_{2}$ within the whole switching period.

Moreover, by using the Agilent E5061B LF-RF network analyzer, bode diagram of $G_{v d}(\mathrm{~s})$ from the circuit experiments is directly obtained, as shown in Fig. 8. Comparing Fig. 8 with the Fig. 4 and Fig. 6, one can see that the circuit experimental results are in good agreement with the PSIM simulations and the theoretical calculations. Therefore, it is confirmed further that the derived control-to-output transfer function $G_{v d}(\mathrm{~s})$ is an effective model for the open-loop stepup converter. In other words, the derived average model and small signal model here are effective for describing the small signal dynamical behaviors of the step-up converter.

\section{Conclusion}

By using the average method and the geometric technique to calculate the average value, the average model and the corresponding small signal model of the open-loop stepup converter are established. The derived DC equilibrium point shows that the DC output voltage includes not only the DC input voltage and the DC duty cycle, but also the two energy-transferring capacitors, the switching frequency and the load. Moreover, the control-to-output transfer function is also derived and its theoretical calculations are in good agreement with the PSIM simulations and the circuit experiments. Therefore, the derived average model, the small signal model and the control-to-output transfer function are effective for the open-loop step-up converter and these will be helpful for designing the step-up converter in practical engineering.

\section{Acknowledgements}

This work was supported by the National Natural Science Foundation of China (Grant No. 51377124, 51007068, 51221005), a Foundation for the Author of National Excellent Doctoral Dissertation of PR China (Grant No. 201337), the Program for New Century Excellent Talents in University of China (Grant No. NCET-13-0457), the Specialized Research Fund for the Doctoral Program of Higher Education of China (Grant No. 20100201120028), the Natural Science Basic Research Plan in Shaanxi Province of China (Grant No. 2012JQ7026), the Fundamental Research Funds for the Central Universities of China (Grant No. 2012 jdgz09).

\section{References}

[1] R. W. Erickson and D. Maksimovic, Fundamentals of Power Electronics (2nd ed), Kluwer Academic Publishers: Boston, MA, 2001.

[2] F. L. Luo and H. Ye, Essential DC/DC Converters. 
Taylor \& Francis: New York, USA, 2006.

[3] L. K. Wong and T. K. Man, "Small signal modeling of open-loop SEPIC converters," IET Power Electron., Vol. 3, No. 6, pp. 858-868, 2010.

[4] D. Kwon and G. A. Rincon-Mora, "Single-inductormultiple-output switching DC-DC converters," IEEE Trans. Circuits Syst. II, Express. Briefs, Vol. 56, No. 8, pp. 614-618, Aug 2009.

[5] K. I. Hwu, K. W. Huang, and W. C. Tu, "Step-up converter combining KY and Buck-Boost converters," Electron. Lett., Vol. 47, No. 12, $9^{\text {th }}$ Jun. 2011.

[6] L. Benadero, V. Moreno-Font, R. Giral, and A. EI Aroudi, "Topologies and control of a class of single inductor multiple-output converters operating in continuous conduction mode," IET Power Electron., Vol. 4, No. 8, pp. 927-935, 2011.

[7] Y. B. Zhao, D. Y. Zhang, and C. J. Zhang, "Study on bifurcation and stability of the closed-loop currentprogrammed Boost converters," Chin. Phys., Vol. 16, No. 4, pp. 933-936, 2007.

[8] K. I. Hwu and Y. T. Yau, "KY converter and its derivatives," IEEE Trans. Power Electron., Vol. 24, No. 1, pp. 128-137, Jan. 2009.

[9] S. K. Changchien, T. J. Liang, J. F. Chen, and L. S. Yang, "Novel high step-up DC-DC converter for fuel cell energy conversion system," IEEE Trans. Ind. Electron., Vol. 57, No. 6, pp. 2007-2017, Jun. 2010.

[10] W. H. Li, Y. Zhao, Y. Deng, and X. N. He, "Interleaved converter with voltage multiplier cell for high step-up and high-effciency conversion," IEEE Trans. Power Electron., Vol. 25, No. 9, pp. 2397-2408, Sep. 2010.

[11] L. S. Yang, T. J. Liang, and J. F. Chen, "Transformerless DC-DC converters with high step-up voltage gain,” IEEE Trans. Ind. Electron., Vol. 56, No. 8, pp. 3144-3152, Aug. 2009.

[12] T. Sammaljarvi, F. Lakhdari, M. Karppanen, and T. Suntio, "Modelling and dynamic characterisation of peak-current-mode-controlled superboost converter," IET Power Electron., Vol. 1, No. 4, pp. 527-536, 2008.

[13] A. Kavitha and G. Uma, "Resonant parametric perturbation method to control chaos in current mode controlled DC-DC Buck-Boost converter," Journal of Electrical Engineering \& Technology, Vol. 5, No. 1, pp. 171-178, 2010.

[14] S. -W. Lee, S. -R. Lee, and C. -H. Jeon, "A new high efficient bi-directional DC/DC converter in the dual voltage system," Journal of Electrical Engineering \& Technology, Vol. 1, No. 3, pp. 343-350, 2006.

[15] M. Zhu and F.L. Luo, "Super-lift DC-DC converters: graphical analysis and modelling," Journal of Power Electronics, Vol. 9, No. 6, pp. 854-865, Nov. 2009.

[16] B.-R. Lin and C. -C. Chen, "New three-level PWM DC/DC converter-analysis, design and experiments," Journal of Power Electronics, Vol. 14, No. 1, pp. 3039, Jan. 2014.

[17] S. Onoda and A. Emadi, "PSIM-based modeling of automotive power systems: conventional, electric, and hybrid electric vehicles," IEEE Trans. Veh. Technol., Vol. 53, No. 2, pp. 390-400, Mar. 2004.

[18] M. Veerachary, "PSIM circuit-oriented simulator model for the nonlinear photovoltaic sources," IEEE Trans. Aerosp. Electron. Syst., Vol. 42, No. 2, pp. 735-740, Apr. 2006.

[19] PSIM User's Guide, Version 9.0, Release 3, Powersim Inc. May 2010.

[20] N. Femia, M. Fortunato, G. Petrone, G. Spagnuolo, and M. Vitelli, "Dynamic model of one-cycle control for converters operating in continuous and discontinuous conduction modes," Int. J. Circ. Theor. Appl., Vol. 37, pp. 661-684, 2009.

[21] R. D. Middlebrook and S. Cuk, "A general unified approach to modeling switching-converter power stages,” Int. J. Electron., Vol. 42, No. 6, pp. 521-550, 1977.

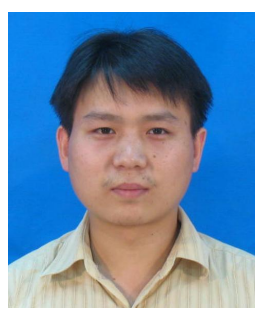

Faqiang Wang was born in China in 1980. He received his B.S. in Automation from Xiangtan University, Xiangtan, China, in 2003, and M.S. and Ph.D. in Electrical Engineering from Xi'an Jiaotong University, Xi'an, China, in 2006 and 2009, respectively. From 2009 to 2011, he was a lecture with School of Electrical Engineering, Xi'an Jiaotong University. Since 2011, he has been an Associate Professor in School of electrical Engineering, Xi'an Jiaotong University. His current research interests include modeling, analysis and control of power electronics.

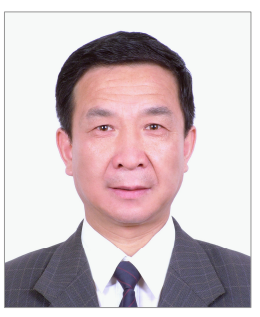

Xikui Ma was born in Shaanxi, China, in 1958. He received the B.S and M.S degrees in electrical engineering from Xi'an Jiaotong University, China, in 1982 and 1985, respectively. Then, he joined the Faculty of Electrical Engineering, Xi' an Jiaotong University as a lecture in 1985, where he became a Professor in 1992. Now, he is the Chair of the Electromagnetic Fields and Microwave Techniques Research Group. During the academic year 1994-1995, he was a visiting scientist at the Power Devices and Systems Research Group, Department of Electrical Engineering and Computer, University of Toronto. His main areas of research interests include electromagnetic field theory and its applications, analytical and numerical methods in solving electromagnetic problems, the field theory of nonlinear materials, modeling of magnetic component, chaotic dynamics and its applications in power electronics, and the applications of digital control to power electronics. 